\title{
Modification of Electromagnetic Field in Photonic Crystal Medium and New Applications of Photonic Band Gap Materials
}

\author{
R.Kh. Gainutdinov ${ }^{1}$ a, M.A. Khamadeev ${ }^{1}$, and M.Kh. Salakhov ${ }^{1,2}$ \\ ${ }^{1}$ Kazan Federal University, Institute of Physics, 18 Kremlevskaya Str. Kazan 420008, Russia \\ ${ }^{2}$ Tatarstan Academy of Sciences, 20 Bauman Str. Kazan 420111, Russia
}

\begin{abstract}
A novel quantumelectrodynamical effect predicted to occur in photonic crystal medium is presented. Influence of the effect on energy levels and spectra of atoms is discussed.
\end{abstract}

Keywords: electron mass, photonic crystal.

Photonic crystal (PC) has received considerable attention mostly because of their possessing photonic band gaps: the ranges of frequency in which a photon cannot propagate in one or more directions. It was shown in [1] that a new type of quantum-electrodynamical effect occurs in photonic crystals when the electron rest mass changes in photonic crystal medium, and this changing depends on the direction of the momentum of an electron. This effect is important not only from the fundamental point of view but also for applications. It opens new ways of using PC's that in contrast to the ordinary ways are not based on the band gap effect. Actually, the effect is another consequence of the periodic changing of the photon-matter interaction in PC medium. Because of this periodicity the contribution of vacuum modes (virtual photons) to atomic-physics processes, that is negligible from the practical point of view (the Lamb shift) in free space, becomes very significant in PC medium. This effect allows one to drive the structure of the atomic energy levels and hence to drive resonance processes.

In [1] it has been shown that because of the anisotropy of PC medium the correction to the energy of a hydrogen-like atom caused by the effect depends on the orbital angular momentum 1 and its $z-$ component $\mathrm{m}$. Moreover, the ionization energy also changes and depends on the direction of the detached electron. In the case of a multi-electron atom the situation becomes more complex. In fact, in the case of the well-known Stark and Zeeman effects corrections to the energy of the states depend only on total quantum numbers such as $\mathrm{J}, \mathrm{L}$ and $\mathrm{S}$ and their projections. It gives us a reason to claim that in the spectra of the atoms placed in PC medium the splitting of a new type should be observed.

\section{Reference}

1. R. Kh. Gainutdinov, M. A. Khamadeev, M. Kh. Salakhov, Phys. Rev. A. 85, 5, 0538362 (2012)

\footnotetext{
${ }^{a}$ Corresponding author: Renat.Gainutdinov@kpfu.ru
} 